\title{
Of adding oranges and apples: how non-abstract representations may foster abstract numerical cognition
}

\author{
Andrea Bender* and Sieghard Beller \\ Department of Psychosocial Science, University of Bergen, Bergen, Norway \\ *Correspondence: andrea.bender@psysp.uib.no \\ Edited by: \\ Philippe Chassy, Liverpool Hope University, UK \\ Reviewed by: \\ Mark Yates, University of Melbourne, Australia \\ Samar Zebian, Lebanese American University, Lebanon
}

Keywords: numerical cognition, number systems, culture, distributed cognition, abstract mathematical thinking

\section{INTRODUCTION}

The realm of numbers constitutes just one of many fields of mathematical cognition, but arguably a pivotal one. It is also among those core domains of knowledge that - while being prepared for unfolding in the human species (Feigenson et al., 2004; Hyde, 2011)—nonetheless requires cultural mediation to unfold to its full potential: Not only is the availability of a conventionalized counting sequence essential for accurate counting and calculating (Gordon, 2004; Pica et al., 2004; Frank et al., 2008; Spaepen et al., 2011), acquiring a counting sequence in the first place is also crucial in more fundamental ways: for grasping the concept of precise quantities, for comprehending the ordinal and cardinal nature of numbers, or for learning the algorithms of basic arithmetics that then pave the way for higher levels of mathematics.

Learning to count also promotes acquaintance with some of the more general principles that characterize mathematics such as abstractness. In fact, one of the first principles to be learned in this process is that numbers are abstractall kinds of entities can be counted with the same number words (Gelman and Gallistel, 1978; but see also Cohen Kadosh and Walsh, 2009). But not all counting sequences seem to reflect this principle. A substantial number of Oceanic languages, for instance, have counting sequences whose usage is restricted to specific objects, while other objects are counted otherwise (Bender and Beller, 2006a,b).

This pattern of counting different things differently seems to directly contradict the abstractness principle and has thus been taken as an earlier stage in the evolution of numerical thinking (e.g., Klix, 1993). While the latter assumption was refuted elsewhere (Beller and Bender, 2008), the question remains open of how (if at all) such apparently non-abstract counting sequences may foster abstract numerical cognition. Here, we defend the position that the Oceanic counting sequences are not only compatible with an abstract understanding of numbers, but may even promote such an understanding. To this end, we propose to conceive of these sequences as the verbal components of the mathematical code, which provide the symbols that people use to represent and manipulate abstract mathematical concepts. Analyzing how the specific properties of these symbol systems affect the processing of numerical information will help us to understand better how abstract mathematical thinking emerges.

\section{COUNTING SEQUENCES AND THEIR COGNITIVE IMPLICATIONS}

In general, each counting sequence consists of a limited set of symbols for basic numbers and (optionally) some composition rules for representing larger numbers. These symbols and composition rules constitute a distinct numeration system, the properties of which may differ substantially across languages (Chrisomalis, 2004; Bender and Beller, 2012; Widom and Schlimm, 2012).

The system's internal structure, for instance, depends on its dimensionality (Zhang and Norman, 1995). Onedimensional systems are unstructured; they either use the same symbol in a cumulative manner to indicate increasing set size (as in tallies), or employ specific symbols distinctively to indicate distinct set sizes (as with the Arabic digits from 1 through 9). In contrast, two-dimensional systems like the English number words make use of a base (in this case: "ten"), which is raised to various powers ("hundred," "thousand," etc.). Number words in between are composed according to the addition and multiplication principle, as in "two hundred and three."

Counting sequences are cultural tools, whose properties may give rise to "representational effects" (Zhang and Norman, 1995), that is, they affect how numerical information is represented and processed (Nickerson, 1988; Fuson, 1990; Miller et al., 1995; Zhang and Wang, 2005; Schlimm and Neth, 2008; Domahs et al., 2010; Beller and Bender, 2011; Krajcsi and Szabo, 2012). An analysis of such representational effects will help us to illuminate the cognitive implications of specific counting systems.

\section{SPECIFIC COUNTING SYSTEMS IN OCEANIC LANGUAGES}

In a large number of Oceanic languages, two types of verbal systems co-exist: regular systems for general counting and systems restricted to counting specific objects in a particular manner (Bender and Beller, 2006a,b). For illustration, take the Polynesian language spoken on Tonga, an island group in the Western Pacific.

Tongan employs five numeration systems: a general and four specific ones. All of them contain primary numerals for the numbers 1 through 10 and for the powers of the base up to $10^{5}$ (Bender and Beller, 2007). The specific systems deviate from each other and from the general system 
in three ways: They make use of diverging counting units; they employ distinct lexemes for some of the powers; and they are applied to only one kind of object each. Accordingly, sugar-cane is counted in pairs, whereas coconuts, pieces of yam for planting, and fish are counted in pairs when few, but in scores when numerous.

It was especially the object-specificity of counting that arrested researchers' interest early-on and nurtured the assumption that speakers of languages like Tongan may lack an abstract concept of number (Klix, 1993). However, viewing this feature in the context of the other two peculiarities allows for a more accurate assessment. It reveals that number word composition in the specific systems remains highly systematic. In fact, the rules for composing number words in the general system require only marginal modifications (namely acknowledgement of the counting unit and the specific power numerals) to generate number words in the specific systems. This structural alignment, together with the older age of the general system, also suggests that the specific systems were deliberately developed out of the general one (Bender and Beller, 2006a,b, 2007).

While the structure of the general counting sequence was retained, the counting unit to which its constituents referred (and hence the value of the counted set) was increased. This transformation of values follows the same principle that is inherently instantiated in two-dimensional systems, namely the multiplication principle for composing larger number words. In these systems, the base and its powers are counted as if they were objects: "three hundreds" is similar to "three baskets." Specific systems carry this abstraction one step further by implicating that the "three hundreds" may refer in fact to "three hundreds of pairs or scores."

Adopting the multiplication principle inherent in power term constructions (like "three hundreds") for the creation of specific counting systems is far from being trivial. It requires a sophisticated understanding of counting insofar, as number words are used now to count not just objects, but other numbers and even abstract counting units. To this end, countability is defined recursively, and in doing so, also paves the way for conceiving of multiplication as an algorithm for mental arithmetics.

What at first glance may look laborious - the recursive extraction of numerical values-can, thus, in fact be cognitively advantageous: It allows for more compact representations, which, in the absence of notation, not only reduces cognitive load (Beller and Bender, 2008), but also increases the speed and correctness of mental arithmetic (Lordahl et al., 1970; Bender and Beller, 2013).

\section{CONCLUSION}

With our analysis we hope to have demonstrated, that the apparently non-abstract representations in Oceanic counting systems have indeed fostered abstract numerical cognition. But beyond this rehabilitation of the specific systems and their users, this "exotic" phenomenon is of more general relevance to the cognitive sciences. It also serves as an instance of the recursive process in which cultural tools and cognitive achievements advance each other and thus as an instance of the "ratchet effect" (Tomasello, 1999; and see Wiese, 2003) of culture more generally, which also highlights the importance of anthropological insights for cognitive science theorizing (Beller et al., 2012). By their mere existence and usage, cultural tools may promote cognitive advancement. Designed to serve one purpose, tools generally have more properties than only those relevant to the task at hand, and these properties may then afford new ways of usage or reasoning (see also Miller and Paredes, 1996; Coolidge and Overmann, 2012). It is this extra value of cultural tools that, in the domain of mathematical cognition, promotes abstract thinking.

\section{ACKNOWLEDGMENTS}

This work was supported by a Heisenberg Fellowship from the Deutsche Forschungsgemeinschaft DFG to Andrea Bender (Be 2451/8-2). We are indebted to Martin Silverman for inspiration.

\section{REFERENCES}

Beller, S., and Bender, A. (2008). The limits of counting: numerical cognition between evolution and culture. Science 319, 213-215. doi: 10.1126/science. 1148345
Beller, S., and Bender, A. (2011). Explicating numerical information: when and how fingers support (or hinder) number comprehension and handling. Front. Psychol. 2:214. doi: 10.3389/fpsyg.2011.00214

Beller, S., Bender, A., and Medin, D. L. (2012). Should anthropology be part of cognitive science? Topics Cogn. Sci. 4, 342-353. doi: 10.1111/j.17568765.2012.01196.x

Bender, A., and Beller, S. (2006a). "Fanciful" or genuine? Bases and high numerals in Polynesian number systems. J. Polyn. Soc. 115, 7-46. Available online at: http://www.jstor.org/stable/20707320

Bender, A., and Beller, S. (2006b). Numeral classifiers and counting systems in Polynesian and Micronesian languages: common roots and cultural adaptations. Ocean. Ling. 45, 380-403. doi: 10.1353/ol.2007.0000

Bender, A., and Beller, S. (2007). Counting in Tongan: the traditional number systems and their cognitive implications. J. Cogn. Cult. 7, 213-239. doi: 10.1163/156853707X208495

Bender, A., and Beller, S. (2012). Nature and culture of finger counting: diversity and representational effects of an embodied cognitive tool. Cognition 124, 156-182. doi: 10.1016/j.cognition.2012.05.005

Bender, A., and Beller, S. (2013). Mangarevan invention of binary steps for easier calculation. Proc. Natl. Acad. Sci. U.S.A. doi: $10.1073 /$ pnas. 1309160110

Chrisomalis, S. (2004). A cognitive typology for numerical notation. Cambr. Archaeol. J. 14, 37-52. doi: 10.1017/S0959774304000034

Cohen Kadosh, R., and Walsh, V. (2009). Numerical representation in the parietal lobes: Abstract or not abstract?. Behav. Brain Sci. 32, 313-328. doi: 10.1017/S0140525X09990938

Coolidge, F. L., and Overmann, K. A. (2012). Numerosity, abstraction, and the emergence of symbolic thinking. Curr. Anthropol. 53, 204-225. doi: $10.1086 / 664818$

Domahs, F., Moeller, K., Huber, S., Willmes, K., and Nuerk, H.C. (2010). Embodied numerosity: Implicit hand-based representations influence symbolic number processing across cultures. Cognition 116, 251-266. doi: 10.1016/j.cognition.2010. 05.007

Feigenson, L., Dehaene, S., and Spelke, E. (2004). Core systems of number. Trends Cogn. Sci. 8, 307-314. doi: 10.1016/j.tics.2004.05.002

Frank, M. C., Everett, D. L., Fedorenko, E., and Gibson, E. (2008). Number as a cognitive technology: evidence from Pirahã language and cognition. Cognition 108, 819-824. doi: 10.1016/j.cognition.2008.04.007

Fuson, K. C. (1990). Conceptual structures for multiunit numbers: implications for learning and teaching multidigit addition, subtraction, and place value. Cogn. Instruct. 7, 343-403. doi: 10.1207/s1532690xci0704_4

Gelman, R., and Gallistel, C. R. (1978). The Child's Understanding of Number. Cambridge: Harvard University Press.

Gordon, P. (2004). Numerical cognition without words: evidence from Amazonia. Science 306, 496-499. doi: 10.1126/science.1094492 
Hyde, D. C. (2011). Two systems of non-symbolic numerical cognition. Front. Psychol. 5:150. doi: 10.3389/fnhum.2011.00150

Klix, F. (1993). Erwachendes Denken [Awakening Thinking]. Heidelberg: Spektrum Akademischer Verlag.

Krajcsi, A., and Szabo, E. (2012). The role of number notation: sign-value notation number processing is easier than place-value. Front. Psychol. 3:463. doi: 10.3389/fpsyg.2012.00463

Lordahl, D. S., Krop, H., and Jacobson, L. I. (1970). Information processing using number systems with bases higher than ten. J. Verb. Learn. Verb. Beh. 9, 155-160. doi: 10.1016/S00225371(70)80044-5

Miller, K. F., and Paredes, D. R. (1996). "On the shoulders of giants: cultural tools and mathematical development," in The Nature of Mathematical Thinking, eds R. J. Sternberg and T. Ben-Zeev (Mahwah, NJ: Erlbaum), 83-117.

Miller, K. F., Smith, C. M., Zhu, J., and Zhang, H. (1995). Preschool origins of cross-national differences in mathematical competence: the role of number-naming systems. Psychol. Sci. 6, 56-60. doi: 10.1111/j.1467-9280.1995. tb00305.x
Nickerson, R. S. (1988). Counting, computing, and the representation of numbers. Hum. Factors 30, 181-199.

Pica, P., Lemer, C., Izard, V., and Dehaene, S. (2004). Exact and approximate arithmetic in an Amazonian indigene group. Science 306, 499-503. doi: 10.1126/science. 1102085

Schlimm, D., and Neth, H. (2008). "Modeling ancient and modern arithmetic practices: addition and multiplication with Arabic and Roman numerals," in Proceedings of the 30th Annual Conference of the Cognitive Science Society, eds B. C. Love, K. McRae, and V. M. Sloutsky (Austin, TX: Cognitive Science Society), 2097-2102.

Spaepen, E., Coppola, M., Spelke, E. S., Carey, S. E., and Goldin-Meadow, S. (2011). Number without a language model. Proc. Natl. Acad. Sci. U.S.A. 108, 3163-3168. doi: 10.1073/pnas. 1015975108

Tomasello, M. (1999). The Cultural Origins of Human Cognition. Cambridge: Harvard University Press.

Widom, T. R., and Schlimm, D. (2012). Methodological reflections on typologies for numerical notations. Sci. Context 25, 155-195. doi: $10.1017 /$ S0269889712000038

Wiese, H. (2003). Numbers, Language, and the Human Mind. Cambridge: Cambridge University Press. doi: 10.1017/CBO9780511486562
Zhang, J., and Norman, D. A. (1995). A representational analysis of numeration systems. Cognition 57, 271-295. doi: 10.1016/0010-0277(95) 00674-3

Zhang, J., and Wang, H. (2005). The effect of external representations on numeric tasks. Quart. J. Exp. Psychol. 58, 817-838. doi: $10.1080 / 02724980443000340$

Received: 29 October 2013; accepted: 10 December 2013; published online: 24 December 2013.

Citation: Bender A and Beller S (2013) Of adding oranges and apples: how non-abstract representations may foster abstract numerical cognition. Front. Hum. Neurosci. 7:903. doi: 10.3389/fnhum.2013.00903

This article was submitted to the journal Frontiers in Human Neuroscience.

Copyright (c) 2013 Bender and Beller. This is an openaccess article distributed under the terms of the Creative Commons Attribution License (CC BY). The use, distribution or reproduction in other forums is permitted, provided the original author(s) or licensor are credited and that the original publication in this journal is cited, in accordance with accepted academic practice. No use, distribution or reproduction is permitted which does not comply with these terms. 